\begin{tabular}{|c|c|c|c|c|c|c|c|c|c|c|c|c|c|}
\hline \multicolumn{2}{|c|}{ Table 7.1} & \multicolumn{12}{|c|}{ Animals slaughtered and meat produced } \\
\hline \multicolumn{14}{|c|}{ Monthly averages, totals for four or five week periods and quarters } \\
\hline & & \multicolumn{7}{|c|}{ Animals slaughtered (thousands) } & \multicolumn{5}{|c|}{ Meat - UK supplies \& Domestic Usage1 (thousand tonnes) } \\
\hline & & \begin{tabular}{|c|} 
Steers, \\
heifers and \\
young bulls
\end{tabular} & $\begin{array}{l}\text { Cows and } \\
\text { adult bulls }\end{array}$ & Calves & $\begin{array}{c}\text { Ewes } \\
\text { and rams }\end{array}$ & $\begin{array}{c}\text { Other } \\
\text { sheep } \\
\text { and lambs }\end{array}$ & $\begin{array}{c}\text { Sows and } \\
\text { adults boars }\end{array}$ & Clean pigs & $\begin{array}{c}\text { Beef } \\
\text { and veal }\end{array}$ & $\begin{array}{l}\text { Mutton } \\
\text { and lamb }\end{array}$ & Pork & $\begin{array}{c}\text { Bacon } \\
\text { and ham }\end{array}$ & Poultry \\
\hline & & BFHA & BFHB & $\mathrm{BFHC}$ & BFHD & BFHE & BFHF & BAKP & BFHK & BFHL & BFHM & JS36 & JYXE \\
\hline 2008 & & $2,028.0$ & 559.0 & 44.0 & $2,342.0$ & $14,401.0$ & 235.0 & $9,193.0$ & $1,060.2$ & 372.4 & 827.5 & 455.0 & $1,558.1$ \\
\hline 2009 & & $2,075.0$ & 509.0 & 43.0 & $2,183.0$ & $13,357.0$ & 211.0 & $9,293.0$ & .. & .. & .. & .. & .. \\
\hline \multirow[t]{4}{*}{2008} & $\operatorname{Sep}^{2}$ & 168.0 & 47.0 & 5.0 & 205.0 & $1,292.0$ & 17.0 & 724.0 & 262.9 & 89.6 & 201.1 & 110.2 & 379.1 \\
\hline & Oct & 204.0 & 64.0 & 6.0 & 239.0 & $1,583.0$ & 21.0 & 903.0 & - & - & - & - & - \\
\hline & Nov & 159.0 & 58.0 & 5.0 & 190.0 & $1,289.0$ & 16.0 & 736.0 & - & - & - & - & - \\
\hline & Dec & 148.0 & 46.0 & 4.0 & 208.0 & $1,343.0$ & 13.0 & 695.0 & 267.1 & 87.2 & 200.5 & 119.3 & 400.3 \\
\hline \multirow[t]{12}{*}{2009} & Jan & 187.0 & 51.0 & 3.0 & 207.0 & $1,260.0$ & 19.0 & 816.0 & - & - & - & - & - \\
\hline & Feb & 159.0 & 39.0 & 3.0 & 170.0 & 909.0 & 16.0 & 674.0 & - & - & - & - & - \\
\hline & Mar & 162.0 & 36.0 & 4.0 & 172.0 & 891.0 & 16.0 & 700.0 & 244.6 & 92.7 & 196.9 & 118.0 & 390.6 \\
\hline & Apr & 199.0 & 41.0 & 3.0 & 201.0 & $1,058.0$ & 19.0 & 834.0 & - & - & - & - & - \\
\hline & May & 161.0 & 35.0 & 2.0 & 150.0 & 762.0 & 15.0 & 675.0 & - & _- & - & - & _- \\
\hline & Jun & 162.0 & 37.0 & 3.0 & 156.0 & 852.0 & 15.0 & 708.0 & 259.8 & 92.7 & 211.0 & 117.0 & 388.7 \\
\hline & Jul & 184.0 & 45.0 & 4.0 & 202.0 & $1,261.0$ & 20.0 & 888.0 & - & - & - & - & - \\
\hline & Aug & 143.0 & 36.0 & 4.0 & 184.0 & $1,207.0$ & 17.0 & 745.0 & - & - & - & - & - \\
\hline & Sep & 162.0 & 42.0 & 4.0 & 173.0 & $1,223.0$ & 17.0 & 739.0 & 251.6 & 84.9 & 200.5 & 110.3 & 372.7 \\
\hline & Oct & 213.0 & 57.0 & 6.0 & 216.0 & $1,547.0$ & 22.0 & 955.0 & .. & .. &.. & .. & .. \\
\hline & Nov & 172.3 & 49.1 & 3.9 & 191.5 & $1,233.3$ & 17.3 & 739.8 & .. & .. & .. & .. & .. \\
\hline & Dec & 157.8 & 40.9 & 3.3 & 152.6 & $1,101.7$ & 15.2 & 721.0 & .. & .. & .. & .. & .. \\
\hline \multirow[t]{8}{*}{2010} & Jan & 186.9 & 48.3 & 2.9 & 150.0 & 959.0 & 20.1 & 844.3 & .. & .. &.. & .. & .. \\
\hline & Feb & 166.6 & 46.4 & 3.5 & 138.5 & 754.4 & 17.7 & 702.2 & .. & .. & .. & .. & .. \\
\hline & Mar & 168.8 & 40.9 & 4.3 & 131.4 & 813.9 & 18.0 & 707.3 & .. & .. &.. & .. & .. \\
\hline & Apr & 207.6 & 46.3 & 4.2 & 161.9 & 919.6 & 21.1 & 858.3 & .. & .. &.. &.. & .. \\
\hline & May & 167.5 & 38.6 & 2.8 & 131.3 & 691.0 & 16.3 & 694.2 & .. & .. & .. & .. & .. \\
\hline & Jun & 166.3 & 38.1 & 3.0 & 137.1 & 755.6 & 15.2 & 685.0 & .. & .. &.. &.. & .. \\
\hline & Jul-02 & 201.6 & 51.8 & 5.0 & 187.1 & $1,200.9$ & - & 855.9 & .. & .. & .. & .. & .. \\
\hline & Aug & 158.3 & 40.3 & 5.1 & 168.9 & $1,120.9$ & - & 717.1 & .. & .. & .. & .. & .. \\
\hline
\end{tabular}

1 From January 2007, 'Meat - UK supplies \& Domestic Usage' are shown as quarters and the data are provisional.

2 Revisions are possible from July 2010.

Source: Department for Environment, Food and Rural Affairs: 01904455803

\begin{tabular}{|c|c|c|c|c|c|c|c|c|c|c|c|c|c|}
\hline \multicolumn{2}{|c|}{ Table 7.2} & \multicolumn{12}{|c|}{ Cereals and cereal products } \\
\hline \multicolumn{12}{|c|}{ Monthly averages or totals for four or five week periods. Stocks refer to the end of the period } & \multicolumn{2}{|c|}{ Thousand tonnes } \\
\hline & & \multicolumn{4}{|c|}{ Wheat } & \multicolumn{2}{|c|}{ Flour } & \multicolumn{4}{|c|}{ Oats } & \multicolumn{2}{|c|}{ Barley } \\
\hline & & \begin{tabular}{c|}
$\begin{array}{c}\text { Homegrown } \\
\text { wheat } \\
\text { milled }\end{array}$ \\
\end{tabular} & \begin{tabular}{c|} 
Imported \\
wheat milled
\end{tabular} & $\begin{array}{c}\text { Home grown } \\
\text { wheat closing } \\
\text { stock }\end{array}$ & $\begin{array}{c}\text { Imported } \\
\text { wheat) closing } \\
\text { stock }\end{array}$ & $\begin{array}{c}\text { Flour } \\
\text { produced }\end{array}$ & $\begin{array}{l}\text { Flour } \\
\text { closing } \\
\text { stocks } \\
\end{array}$ & $\begin{array}{l}\text { Oats } \\
\text { milled }\end{array}$ & $\begin{array}{c}\text { Gross } \\
\text { production }\end{array}$ & Stocks & $\begin{array}{l}\text { Total } \\
\text { barley } \\
\text { usage } \\
\end{array}$ & $\begin{array}{c}\text { Barley } \\
\text { used for } \\
\text { malting }\end{array}$ & Stocks \\
\hline 2006 & & BFDB & BFDC & JSG6 & JSG7 & BFDE & JSG8 & $\mathrm{BFDH}$ & JSG9 & BFDJ & JSH2 & JSH4 & BFDM \\
\hline $\begin{array}{l}2007 \\
2008\end{array}$ & & $\begin{array}{l}391 \\
410\end{array}$ & $\begin{array}{r}82 \\
100\end{array}$ & $\begin{array}{l}182 \\
182\end{array}$ & $\begin{array}{l}104 \\
140\end{array}$ & $\begin{array}{l}384 \\
404\end{array}$ & $\begin{array}{l}58 \\
58\end{array}$ & $\begin{array}{l}35 \\
35\end{array}$ & $\begin{array}{l}21 \\
21\end{array}$ & $\begin{array}{l}19 \\
23\end{array}$ & $\begin{array}{l}144 \\
148\end{array}$ & $\begin{array}{l}139 \\
140\end{array}$ & $\begin{array}{l}698 \\
741\end{array}$ \\
\hline 2007 & Q4 & 389 & 105 & 169 & 143 & 388 & 57 & 35 & 21 & 16 & 144 & 138 & 925 \\
\hline 2008 & $\begin{array}{l}\text { Q1 } \\
\text { Q2 } \\
\text { Q3 } \\
\text { Q4 }\end{array}$ & $\begin{array}{l}402 \\
407 \\
413 \\
417\end{array}$ & $\begin{array}{r}101 \\
107 \\
102 \\
91\end{array}$ & $\begin{array}{l}196 \\
213 \\
157 \\
163\end{array}$ & $\begin{array}{l}181 \\
148 \\
115 \\
115\end{array}$ & $\begin{array}{l}395 \\
407 \\
408 \\
406\end{array}$ & $\begin{array}{l}59 \\
55 \\
58 \\
59\end{array}$ & $\begin{array}{l}35 \\
37 \\
34 \\
35\end{array}$ & $\begin{array}{l}21 \\
22 \\
20 \\
21\end{array}$ & $\begin{array}{l}24 \\
27 \\
21 \\
19\end{array}$ & $\begin{array}{l}148 \\
148 \\
145 \\
151\end{array}$ & $\begin{array}{l}141 \\
140 \\
138 \\
144\end{array}$ & $\begin{array}{l}780 \\
574 \\
614 \\
997\end{array}$ \\
\hline 2009 & $\begin{array}{l}\text { Q1 } \\
\text { Q2 } \\
\text { Q3 }\end{array}$ & $\begin{array}{l}398 \\
405 \\
409\end{array}$ & $\begin{array}{r}97 \\
110 \\
105\end{array}$ & $\begin{array}{l}167 \\
172 \\
157\end{array}$ & $\begin{array}{l}115 \\
116 \\
118\end{array}$ & $\begin{array}{l}395 \\
412 \\
411\end{array}$ & $\begin{array}{l}61 \\
59 \\
58\end{array}$ & $\begin{array}{l}37 \\
34 \\
. .\end{array}$ & $\begin{array}{l}22 \\
21 \\
. .\end{array}$ & $\begin{array}{l}21 \\
20 \\
. .\end{array}$ & $\begin{array}{l}152 \\
138 \\
120\end{array}$ & $\begin{array}{l}145 \\
131 \\
113\end{array}$ & $\begin{array}{l}881 \\
681 \\
779\end{array}$ \\
\hline 2008 & $\mathrm{Dec}^{4}$ & 421 & 101 & 182 & 111 & 421 & 59 & 38 & 23 & 17 & 155 & 148 & 988 \\
\hline 2009 & $\begin{array}{l}\text { Jan } \\
\text { Feb }\end{array}$ & $\begin{array}{l}359 \\
376\end{array}$ & $\begin{array}{l}88 \\
90\end{array}$ & $\begin{array}{l}169 \\
167\end{array}$ & $\begin{array}{l}116 \\
113\end{array}$ & $\begin{array}{l}353 \\
374\end{array}$ & $\begin{array}{l}64 \\
60\end{array}$ & $\begin{array}{l}31 \\
38\end{array}$ & $\begin{array}{l}18 \\
23\end{array}$ & $\begin{array}{l}17 \\
22\end{array}$ & $\begin{array}{l}152 \\
141\end{array}$ & $\begin{array}{l}146 \\
136\end{array}$ & $\begin{array}{l}921 \\
885\end{array}$ \\
\hline & Mar & 457 & 112 & 165 & 116 & 457 & 60 & 42 & 25 & 23 & 163 & 154 & 838 \\
\hline & Apr & 376 & 101 & 168 & 99 & 383 & 61 & 31 & 19 & 20 & 138 & 134 & 770 \\
\hline & May & 390 & 104 & 165 & 126 & 394 & 59 & 33 & 21 & 17 & 135 & 128 & 680 \\
\hline & Jun & 447 & 126 & 173 & 123 & 459 & 57 & 37 & 23 & 23 & 140 & 132 & 592 \\
\hline & Jul & 398 & 106 & 170 & 115 & 402 & 57 & .. & .. & .. & 120 & 114 & 556 \\
\hline & Aug & 385 & 102 & 147 & 126 & 389 & 58 & .. & .. & .. & 112 & 105 & 749 \\
\hline & Sep & 443 & 107 & 154 & 112 & 441 & 58 & .. & .. & .. & 129 & 121 & 1,032 \\
\hline & Oct & 395 & 84 & 162 & 105 & 383 & 56 &.. & .. & .. & 135 & 127 & 1,023 \\
\hline & Nov & 398 & 82 & 172 & 98 & 384 & 57 & .. & .. & .. & 136 & 129 & 1,034 \\
\hline & Sep & 447 & 441 & 83 & 550 & 415 & 414 & 41 & 37 & 21 & 44 & 438 & 218 \\
\hline & Oct & 412 & 397 & 71 & 663 & 367 & 371 & 31 & 32 & 18 & 45 & 209 & 213 \\
\hline & Nov & 386 & 378 & 72 & 384 & 357 & 359 & 34 & 35 & 21 & 20 & 223 & 229 \\
\hline & Dec & 439 & 429 & 82 & 402 & 405 & 405 & 33 & 36 & 20 & 17 & 223 & 252 \\
\hline
\end{tabular}

1 Stocks held by wheat millers, feed compounders, cereal breakfast food manufacturers, brewers, maltsters and distillers, merchants and dealers.

2 Sales of UK grown barley to brewers, maltsters and distillers.

3 Other than oatmeal and oatmeal flakes.

4 Revisions are possible from December 2008.

Source: Department for Environment, Food and Rural Affairs: 01904455803 
Thousand tonnes

\begin{tabular}{|c|c|c|c|c|c|c|c|c|}
\hline & & $\begin{array}{l}\text { Cattle and } \\
\text { calf feed }\end{array}$ & Pig feed & Poultry feed & Sheep feed & Horse feed & Other compounds & Total \\
\hline & & BFFB & BFFD & BFFE & JJ8R & JJ8S & BFFF & BFFA \\
\hline \multirow{11}{*}{2007} & Feb & 333.9 & 120.7 & 206.0 & 110.4 & 17.4 & 21.5 & 810.0 \\
\hline & Mar & 384.4 & 139.3 & 248.2 & 153.6 & 19.9 & 21.5 & 966.9 \\
\hline & Apr & 305.0 & 123.2 & 217.4 & 79.6 & 14.8 & 22.5 & 762.4 \\
\hline & May & 239.2 & 126.8 & 230.7 & 32.1 & 13.4 & 26.5 & 668.7 \\
\hline & Jun & 249.1 & 145.0 & 269.9 & 25.1 & 11.9 & 33.0 & 734.0 \\
\hline & Jul & 268.8 & 133.0 & 259.2 & 21.1 & 12.5 & 34.0 & 728.6 \\
\hline & Aug & 294.2 & 134.5 & 251.8 & 25.1 & 13.0 & 36.2 & 754.7 \\
\hline & Sep & 322.6 & 147.1 & 253.8 & 25.0 & 15.7 & 36.3 & 800.6 \\
\hline & Oct & 332.1 & 137.7 & 237.1 & 24.5 & 15.9 & 30.2 & 777.6 \\
\hline & Nov & 341.3 & 132.2 & 237.4 & 31.8 & 18.5 & 25.9 & 787.2 \\
\hline & Dec & 395.2 & 144.2 & 253.8 & 51.8 & 20.6 & 25.3 & 890.9 \\
\hline \multirow[t]{12}{*}{2008} & Jan & 352.8 & 123.6 & 222.5 & 84.5 & 20.2 & 21.8 & 825.5 \\
\hline & Feb & 353.5 & 116.2 & 215.7 & 109.3 & 18.7 & 22.7 & 836.1 \\
\hline & Mar & 392.1 & 132.5 & 248.2 & 142.3 & 20.3 & 24.4 & 959.8 \\
\hline & Apr & 368.7 & 118.3 & 225.2 & 115.3 & 19.2 & 21.8 & 868.4 \\
\hline & May & 245.1 & 114.9 & 231.9 & 29.9 & 12.8 & 27.4 & 662.0 \\
\hline & Jun & 250.6 & 125.9 & 270.7 & 23.1 & 11.5 & 25.6 & 707.4 \\
\hline & Jul ${ }^{1}$ & 256.8 & 119.0 & 265.2 & 18.6 & 11.9 & 28.2 & 699.6 \\
\hline & Aug & 262.9 & 115.1 & 240.1 & 17.4 & 11.6 & 31.9 & 679.0 \\
\hline & Sep & 319.9 & 130.7 & 263.8 & 26.0 & 16.9 & 38.3 & 795.5 \\
\hline & Oct & 333.9 & 118.7 & 238.8 & 31.6 & 15.8 & 32.9 & 771.9 \\
\hline & Nov & 321.2 & 112.8 & 227.5 & 43.7 & 16.7 & 27.1 & 749.0 \\
\hline & Dec & 382.9 & 128.9 & 251.4 & 63.6 & 21.6 & 27.5 & 875.9 \\
\hline \multirow[t]{5}{*}{2009} & Jan & 327.3 & 104.7 & 210.8 & 91.3 & 17.8 & 24.9 & 776.8 \\
\hline & Feb & 315.5 & 101.8 & 198.7 & 112.8 & 17.2 & 23.8 & 769.9 \\
\hline & Mar & 369.5 & 116.9 & 242.6 & 142.1 & 19.1 & 18.7 & 908.9 \\
\hline & Apr & 295.4 & 106.4 & 221.2 & 82.6 & 15.7 & 24.5 & 745.8 \\
\hline & May & 249.4 & 105.4 & 229.8 & 36.4 & 11.8 & 27.8 & 660.5 \\
\hline
\end{tabular}

1 Revisions are possible from July 2008.

Source: Department for Environment, Food and Rural Affairs: 01904455803

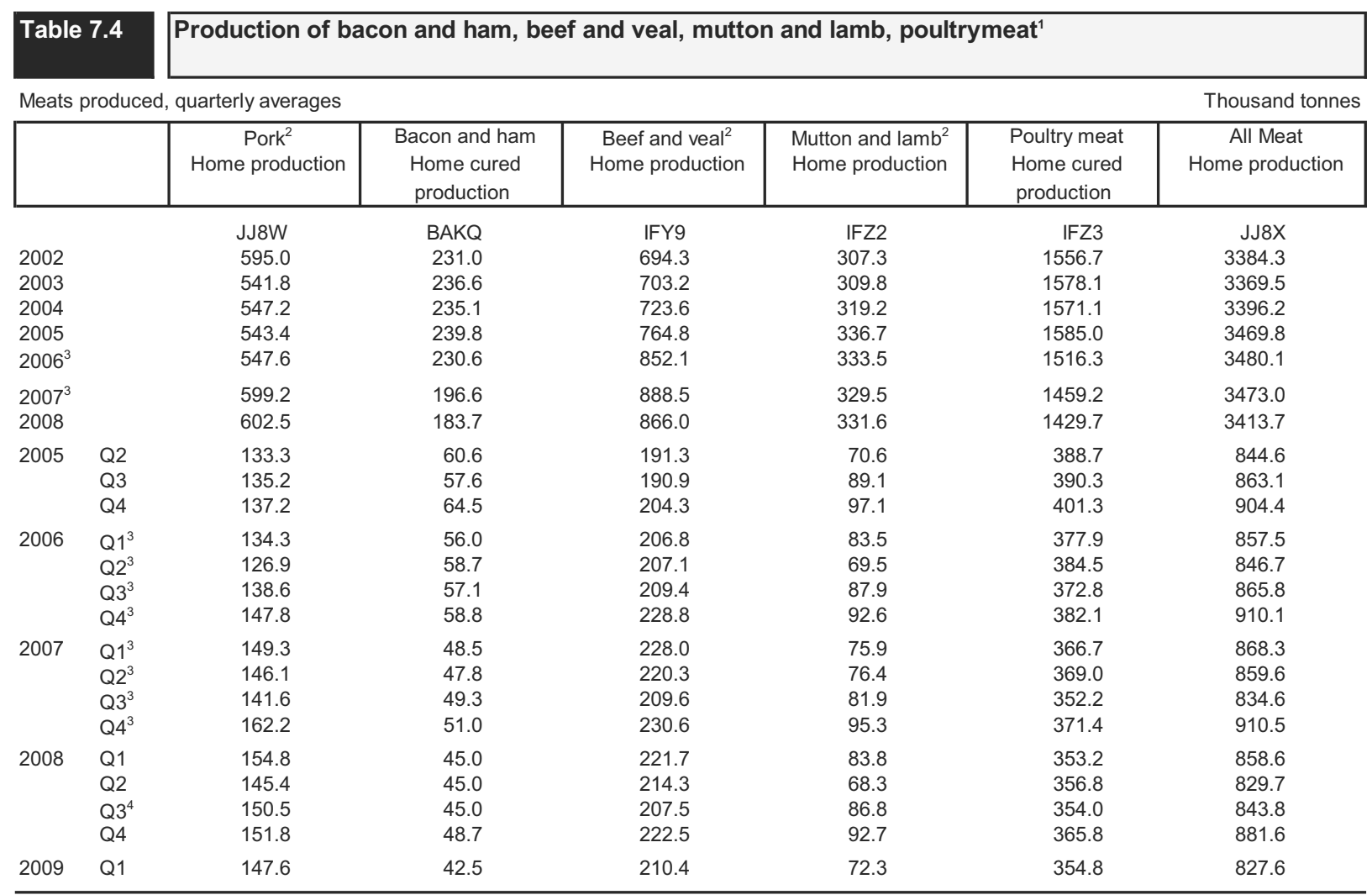

1 Does not include meat offals or trade in preserved or manufactured meat products (eg: canned meat).

2 Includes an estimate of the dressed carcase weight of live exports other than pure bred.

3 Figures have been rounded individually and totals will not necessarily agree with the addition of separate figures.

Source: Department for Environment, Food and Rural Affairs: 01904455803 


\begin{tabular}{|c|c|c|c|c|c|c|c|c|c|}
\hline \multicolumn{2}{|c|}{ Table 7.5} & \multicolumn{8}{|c|}{ Milk and milk products } \\
\hline \multicolumn{10}{|c|}{ Monthly averages or calendar months } \\
\hline & & \multicolumn{4}{|c|}{ Million litres } & \multicolumn{4}{|c|}{ Thousand tonnes } \\
\hline & & \multicolumn{4}{|c|}{ Disposals of raw milk for manufacture } & \multicolumn{4}{|c|}{ Wholesale production ${ }^{2}$} \\
\hline & & $\begin{array}{l}\text { Liquid } \\
\text { milk }^{1}\end{array}$ & Total & Exports & $\begin{array}{l}\text { Stock change } \\
\& \text { wastage }\end{array}$ & Condensed milk ${ }^{3}$ & Milk powders ${ }^{3,4}$ & Butter & Cheese \\
\hline & & IFX4 & IFX5 & IFX6 & IFX7 & IFX8 & IFX9 & IFY2 & IFY3 \\
\hline 2004 & & $6,693.3$ & $6,723.9$ & 434.3 & 106.9 & 161.2 & 167.6 & 249.4 & $3,401.2$ \\
\hline 2005 & & $6,652.5$ & $6,489.8$ & 626.3 & 86.1 & 142.7 & 121.5 & 266.5 & $3,705.4$ \\
\hline 2006 & & $6,734.3$ & $6,266.3$ & 617.3 & 99.7 & 113.3 & 114.1 & 241.2 & $3,778.9$ \\
\hline 2007 & & $6,723.7$ & $6,085.1$ & 537.6 & 126.8 & 109.2 & 117.0 & 248.1 & $3,561.0$ \\
\hline 2008 & & $6,678.3$ & $5,840.0$ & 559.2 & 91.5 & 109.9 & 94.3 & 228.9 & $3,635.4$ \\
\hline 2009 & & $6,626.4$ & $5,699.4$ & 433.4 & 293.4 & 104.3 & 101.7 & 241.6 & $3,369.0$ \\
\hline \multirow[t]{4}{*}{2008} & Sep & 542.0 & 413.8 & 42.8 & -8.3 & 26.0 & 19.8 & 19.0 & 270.2 \\
\hline & Oct & 570.7 & 408.5 & 55.6 & -0.8 & .. & .. & 19.9 & 279.8 \\
\hline & Nov & 565.7 & 384.3 & 53.0 & 4.0 & & 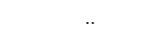 & 18.3 & 249.2 \\
\hline & Dec & 579.9 & 456.7 & 38.1 & -5.8 & 28.3 & 14.4 & 17.3 & 271.9 \\
\hline \multirow[t]{12}{*}{2009} & Jan & 560.6 & 460.3 & 38.5 & 30.8 & .. & .. & 22.5 & 279.6 \\
\hline & Feb & 513.8 & 430.0 & 34.4 & 21.2 & & & 22.4 & 245.2 \\
\hline & Mar & 571.1 & 500.9 & 41.2 & 29.9 & 26.0 & 27.0 & 23.6 & 295.9 \\
\hline & Apr & 548.9 & 542.6 & 41.7 & 26.0 & .. & .. & 23.0 & 317.4 \\
\hline & May & 569.2 & 578.5 & 41.2 & 32.0 & & & 23.9 & 333.4 \\
\hline & Jun & 538.4 & 547.0 & 36.4 & 22.9 & 25.5 & 35.0 & 21.1 & 327.9 \\
\hline & Jul & 567.1 & 489.9 & 41.9 & 18.5 & .. & .. & 19.2 & 299.5 \\
\hline & Aug & 554.4 & 462.3 & 33.4 & 19.2 & .. & .. & 17.7 & 288.3 \\
\hline & Sep & 558.1 & 412.6 & 29.2 & 8.4 & 25.1 & 19.5 & 15.7 & 255.2 \\
\hline & Oct & 553.7 & 427.9 & 29.2 & 27.3 &.. & .. & 16.7 & 258.8 \\
\hline & Nov & 536.1 & 399.6 & 32.6 & 30.0 & .. & .. & 18.1 & 221.0 \\
\hline & Dec & 555.0 & 448.0 & 33.7 & 27.2 & 27.8 & 20.2 & 17.7 & 246.7 \\
\hline \multirow[t]{7}{*}{2010} & $\operatorname{Jan}^{5}$ & 582.5 & 443.2 & 36.2 & 2.8 & .. & .. & 21.3 & 258.5 \\
\hline & $\mathrm{Feb}^{5}$ & 526.5 & 428.9 & 33.7 & 2.1 & .. & .. & 20.2 & 261.9 \\
\hline & $\mathrm{Mar}^{5}$ & 588.4 & 499.4 & 36.5 & 8.0 & $22 . \ddot{4}$ & 20.7 & 20.6 & 304.7 \\
\hline & $\mathrm{Apr}^{5}$ & 550.2 & 548.9 & 38.0 & 11.5 & .. & .. & 22.9 & 321.3 \\
\hline & $\mathrm{Mav}^{5}$ & 596.3 & 617.2 & 37.5 & 9.4 & .. & & 23.9 & 344.0 \\
\hline & $\mathrm{Jun}^{5}$ & 556.8 & 597.8 & 33.0 & 6.9 & 21.2 & 51.8 & 23.8 & 349.1 \\
\hline & $\mathrm{Jul}^{5,6}$ & 583.6 & 527.2 & 36.5 & 8.0 & .. & .. & 19.8 & 308.5 \\
\hline
\end{tabular}

1 Liquid milk that leaves the primary dairy in liquid form. This may be used for manufacture of other food products, or as liquid drinking milk.

2 Excludes production by direct sellers of liquid milk, butter and cheese (ie. Farmhouse production).

3 Monthly figures are not available from 2006 onwards due to confidentiality issues.

4 Full cream, whole milk, partially skimmed milk and skimmed milk powders.

5 Provisional data, may be subject to revision.

6 Revisions are possible from July 2010

Source: Department for Environment Food and Rural Affairs: 01904455803

\begin{tabular}{|c|c|c|c|c|c|c|c|c|}
\hline \multicolumn{2}{|c|}{ Table 7.6} & \multicolumn{7}{|c|}{ Tobacco products released for home consumption } \\
\hline \multicolumn{9}{|c|}{ Monthly averages or calendar months } \\
\hline & & \multirow{2}{*}{\multicolumn{3}{|c|}{$\begin{array}{c}\text { Million } \\
\text { Cigarettes }\end{array}$}} & \multicolumn{4}{|c|}{ Thousand kilogrammes } \\
\hline & & & & & \multicolumn{3}{|c|}{ Other tobacco products } & \multirow{2}{*}{$\begin{array}{l}\text { Total tobacco } \\
\text { products other } \\
\text { than cigarettes }\end{array}$} \\
\hline & & Home-produced & Imported & Total & Cigars & Hand-rolling & Other $^{1}$ & \\
\hline & & LUQN & LUQO & LUQP & LUQQ & LUQR & LUQS & LUQT \\
\hline 2005 & & 45,922 & 4,322 & 50,244 & 758 & 3,189 & 499 & 4,445 \\
\hline 2006 & & 44,392 & 4,570 & 48,962 & 689 & 3,454 & 439 & 4,581 \\
\hline 2007 & & 41,955 & 3,794 & 45,749 & 602 & 3,644 & 398 & 4,643 \\
\hline 2008 & & 42,053 & 3,680 & 45,733 & 546 & 4,154 & 381 & 5,081 \\
\hline 2009 & & 43,989 & 3,586 & 47,575 & 534 & 5,084 & 397 & 6,014 \\
\hline \multirow[t]{3}{*}{2008} & Oct & 3,604 & 308 & 3,912 & 47 & 338 & 33 & 417 \\
\hline & Nov & 3,085 & 328 & 3,413 & 43 & 320 & 27 & 390 \\
\hline & Dec & 3,430 & 341 & 3,771 & 60 & 389 & 34 & 483 \\
\hline \multirow[t]{12}{*}{2009} & $\operatorname{Jan}^{2}$ & 4,224 & 281 & 4,505 & 38 & 336 & 28 & 402 \\
\hline & Feb & 2,846 & 237 & 3,084 & 39 & 393 & 31 & 462 \\
\hline & Mar & 3,305 & 290 & 3,595 & 35 & 415 & 30 & 480 \\
\hline & Apr & 6,902 & 665 & 7,567 & 107 & 801 & 51 & 959 \\
\hline & May & 1,056 & 7 & 1,063 & 18 & 149 & 18 & 185 \\
\hline & Jun & 2,757 & 239 & 2,996 & 39 & 316 & 30 & 385 \\
\hline & Jul & 3,701 & 320 & 4,021 & 34 & 440 & 32 & 506 \\
\hline & Aug & 3,495 & 296 & 3,791 & 43 & 410 & 30 & 483 \\
\hline & Sep & 4,114 & 291 & 4,405 & 44 & 473 & 35 & 552 \\
\hline & Oct & 3,479 & 283 & 3,761 & 42 & 368 & 33 & 443 \\
\hline & Nov & 3,339 & 283 & 3,622 & 50 & 431 & 37 & 519 \\
\hline & Dec & 4,771 & 395 & 5,166 & 45 & 550 & 42 & 637 \\
\hline \multirow[t]{7}{*}{2010} & Jan & 3,512 & 336 & 3,848 & 27 & 320 & 18 & 365 \\
\hline & Feb & 2,028 & 121 & 2,149 & 54 & 439 & 28 & 521 \\
\hline & Mar & 5,686 & 1,023 & 6,708 & 65 & 954 & 61 & 1,081 \\
\hline & Apr & 1125 & 50 & 1175 & 12 & 124 & 14 & 150 \\
\hline & May & 2973 & 255 & 3229 & 50 & 290 & 23 & 363 \\
\hline & Jun $^{3}$ & 5846 & 486 & 6332 & 47 & 718 & 42 & 807 \\
\hline & $\mathrm{Jul}^{3,4}$ & 1801 & 122 & 1923 & 25 & 199 & 24 & 248 \\
\hline
\end{tabular}

Note: Releases of cigarettes and other tobacco products tend to be higher in the period before a Budget.

Products may then be stocks, duty paid, before being sold.

1 Excluding snuff.

2 Due to the effect of the holiday period, these figures are subject to greater uncertainty than usual.

3 Provisional.

4 Revisions are possible from July 2010.

Sources: HM Revenueand Customs Statistical Bulletins at http://www.uktradeinfo.com/index.cfm?task=bulletins 


\begin{tabular}{|c|c|c|c|c|c|c|c|c|c|c|c|c|c|c|c|}
\hline & \multicolumn{9}{|c|}{ Thousand hectolitres } & \multicolumn{5}{|c|}{ Thousand hectolitres of alcohol } \\
\hline & & \multirow[t]{4}{*}{\begin{tabular}{c|} 
Beer \\
production
\end{tabular}} & \multicolumn{8}{|c|}{$\begin{array}{l}\text { Released for home } \\
\text { consumption }\end{array}$} & \multirow{2}{*}{\multicolumn{3}{|c|}{$\begin{array}{c}\begin{array}{c}\text { Released for home } \\
\text { consumption }\end{array} \\
\text { Spirits }\end{array}$}} & \multicolumn{2}{|c|}{$\begin{array}{c}\text { Production of } \\
\text { potable spirits }^{1}\end{array}$} \\
\hline & & & \multirow[t]{3}{*}{ Beer } & \multicolumn{4}{|c|}{ Wine of fresh grapes } & \multicolumn{2}{|c|}{ Made wine } & \multirow{3}{*}{$\begin{array}{c}\text { Cider } \\
\text { and perry }\end{array}$} & & & & \multirow{3}{*}{$\begin{array}{l}\text { Home- } \\
\text { produced } \\
\text { whisky }\end{array}$} & \multirow[t]{3}{*}{ Other } \\
\hline & & & & \multicolumn{2}{|c|}{ Not exceeding $15 \%$} & \multirow{2}{*}{$\begin{array}{c}\text { Over } \\
15 \% \text { ABV }\end{array}$} & \multirow[t]{2}{*}{ Total } & \multirow[t]{2}{*}{ Coolers $^{3}$} & \multirow[t]{2}{*}{ Other } & & & Spirit- & Other $^{5}$ & & \\
\hline & & & & Still & Sparkling & & & & & & $\begin{array}{c}\text { produced } \\
\text { whisky }\end{array}$ & $\begin{array}{c}\text { based } \\
\text { coolers }^{4}\end{array}$ & & & \\
\hline & & BFNK & BAYL & BFNO & BFNS & BFNP & BFNT & BAYM & BAYN & BFNW & BFNX & YZUJ & BFNY & BAYO & BAYP \\
\hline 2006 & & 53768 & 55751 & 11655.4 & 715.0 & 301.5 & 12671.9 & 527.6 & 316.7 & 7522.6 & 282.6 & 64.9 & 766.9 & 3985.7 & 499.5 \\
\hline 2007 & & 51341 & 53465 & 12559.1 & 838.1 & 305.0 & 13702.2 & 720.1 & 348.0 & 8046.0 & 285.9 & 51.8 & 832.2 & 5059.5 & 439.0 \\
\hline 2008 & & 49611 & 51498 & 12402.2 & 757.4 & 323.9 & 13483.5 & 611.5 & 381.5 & 8412.3 & 289.4 & 41.7 & 816.6 & 5516.1 & 556.1 \\
\hline 2009 & & 45141 & 46817 & 11729.3 & 731.3 & 219.1 & 12679.8 & 596.8 & 392.5 & 9403.8 & 258.1 & 31.8 & 801.6 & 4855.0 & 901.8 \\
\hline & & BFNK & BAYL & BFNO & BFNS & BFNP & BFNT & BAYM & BAYN & BFNW & BFNX & YZUJ & BFNY & BAYO & BAYP \\
\hline 2006 & Jan & 3564 & 3157 & 693.6 & 46.9 & 11.5 & 751.9 & 24.8 & 19.7 & 359.4 & 13.0 & 3.3 & 32.2 & & \\
\hline & Feb & 3105 & 3748 & 843.4 & 41.2 & 14.0 & 898.6 & 31.6 & 16.6 & 437.8 & 16.0 & 3.7 & 42.9 & & \\
\hline & Mar & 5218 & 5492 & 1095.5 & 41.4 & 18.9 & 1155.8 & 38.1 & 31.9 & 527.8 & 21.6 & 5.0 & 59.4 & 1019.7 & 140.3 \\
\hline & Apr & 4029 & 4005 & 955.0 & 40.5 & 16.9 & 1012.5 & 42.8 & 19.2 & 560.1 & 21.8 & 5.6 & 59.8 & & \\
\hline & May & 4784 & 5395 & 891.6 & 46.5 & 15.9 & 954 & 35.2 & 22.2 & 647.9 & 20.1 & 5.1 & 55.0 & & \\
\hline & Jun & 5295 & 5362 & 961.7 & 54.9 & 17.8 & 1034.4 & 49.8 & 25.6 & 695.7 & 24.2 & 8.2 & 75.0 & 1222.8 & 118.1 \\
\hline & Jul & 4852 & 4558 & 980.1 & 57.4 & 13.8 & 1051.4 & 50.1 & 24.2 & 753.5 & 16.8 & 4.5 & 50.5 & & \\
\hline & Aug & 4573 & 4900 & 999.3 & 49.7 & 17.0 & 1066 & 49.6 & 25.2 & 849.8 & 21.0 & 7.0 & 64.4 & & \\
\hline & Sep & 4205 & 4332 & 969.1 & 53.8 & 21.6 & 1044.5 & 39.8 & 27.7 & 690.1 & 20.9 & 4.5 & 61.9 & 909.4 & 110.4 \\
\hline & Oct & 4667 & 4740 & 974.9 & 65.7 & 34.8 & 1075.5 & 47.6 & 38.1 & 682.5 & 31.6 & 5.7 & 72.8 & & \\
\hline & Nov & 4053 & 4436 & 1242.6 & 106.9 & 67.5 & 1417 & 53.7 & 42.9 & 695.3 & 38.2 & 4.9 & 100.5 & & \\
\hline & $\mathrm{Dec}^{6}$ & 5423 & 5626 & 1048.5 & 110.1 & 51.8 & 1210.4 & 64.6 & 23.5 & 622.8 & 37.4 & 7.3 & 92.5 & 833.8 & 130.7 \\
\hline 2007 & $\operatorname{Jan}^{6}$ & 3241 & 3221 & 936.1 & 65.0 & 20.1 & 1021.3 & 41.7 & 22.5 & 520.1 & 19.1 & 4.5 & 61.5 & & \\
\hline & Feb & 3303 & 3530 & 821.5 & 40.3 & 11.8 & 873.7 & 36.8 & 18.2 & 495.3 & 15.7 & 3.1 & 48.5 & & \\
\hline & Mar & 4926 & 5342 & 1059.6 & 56.1 & 16.7 & 1132.4 & 54.4 & 35.7 & 650.1 & 21.6 & 3.9 & 59.6 & 1265.5 & 75.2 \\
\hline & Apr & 4135 & 4092 & 979.5 & 51.0 & 18.9 & 1049.4 & 57.1 & 19.8 & 615.6 & 18.9 & 4.6 & 56.6 & & \\
\hline & May & 4494 & 4903 & 1014.9 & 48.9 & 15.2 & 1079.1 & 56.2 & 26.6 & 726.1 & 21.4 & 4.1 & 63.6 & & \\
\hline & Jun & 4278 & 4387 & 1110.9 & 64.1 & 18.3 & 1193.4 & 71.1 & 23.4 & 797.0 & 22.9 & 4.5 & 65.3 & 1359.8 & 91.8 \\
\hline & Jul & 4388 & 4180 & 987.7 & 60.0 & 17.1 & 1064.8 & 53.9 & 30.3 & 608.0 & 18.5 & 2.9 & 54.8 & & \\
\hline & Aug & 4643 & 5307 & 1263.9 & 66.9 & 20.8 & 1351.6 & 79.9 & 32.0 & 760.5 & 27.2 & 6.3 & 78.0 & & \\
\hline & Sep & 4056 & 4057 & 991.0 & 68.5 & 25.3 & 1084.9 & 55.6 & 29.0 & 852.2 & 20.3 & 4.1 & 60.1 & 1181.0 & 94.1 \\
\hline & Oct & 4727 & 4892 & 1061.0 & 74.4 & 34.0 & 1169.5 & 60.3 & 35.1 & 614.2 & 26.3 & 2.6 & 66.6 & & \\
\hline & Nov & 4635 & 4865 & 1325.3 & 128.2 & 55.9 & 1509.4 & 91.7 & 42.6 & 740.2 & 40.9 & 6.4 & 112.6 & & \\
\hline & $\operatorname{Dec}^{6}$ & 4515 & 4689 & 1007.7 & 114.5 & 50.6 & 1172.9 & 61.4 & 32.7 & 666.7 & 33.0 & 4.8 & 105.1 & 1253.2 & 177.8 \\
\hline 2008 & $\operatorname{Jan}^{6}$ & 2998 & 3208 & 772.5 & 51.5 & 20.1 & 844.1 & 26.9 & 22.9 & 547.6 & 15.3 & 2.0 & 41.8 & & \\
\hline & $\mathrm{Feb}$ & 3589 & 3878 & 927.4 & 58.5 & 20.5 & 1006.3 & 37.6 & 23.7 & 513.3 & 17.3 & 2.9 & 46.8 & & \\
\hline & Mar & 4214 & 4462 & 1301.1 & 59.4 & 27.2 & 1387.7 & 49.9 & 48.1 & 780.6 & 34.1 & 4.0 & 90.7 & 1344.6 & 71.3 \\
\hline & Apr & 4884 & 4066 & 800.6 & 39.0 & 14.8 & 854.5 & 41.8 & 18.9 & 542.9 & 10.5 & 1.6 & 43.3 & & \\
\hline & May & 4486 & 4770 & 1103.6 & 57.8 & 37.1 & 1198.6 & 59.1 & 29.6 & 995.4 & 24.4 & 5.5 & 72.6 & & \\
\hline & Jun & 4290 & 4355 & 967.2 & 58.8 & 7.3 & 1033.3 & 42.5 & 30.8 & 619.2 & 19.2 & 3.5 & 60.3 & 1500.4 & 175.0 \\
\hline & Jul & 4082 & 4264 & 1032.5 & 64.9 & 19.2 & 1116.6 & 50.8 & 27.3 & 798.1 & 17.0 & 3.3 & 53.8 & & \\
\hline & Aug & 4233 & 4241 & 1073.7 & 46.3 & 20.6 & 1140.6 & 56.5 & 29.2 & 947.4 & 23.0 & 3.5 & 66.2 & & \\
\hline & Sep & 3909 & 4353 & 834.6 & 43.5 & 15.2 & 893.3 & 42.6 & 27.7 & 480.5 & 21.2 & 2.9 & 58.8 & 1250.4 & 151.2 \\
\hline & Oct & 4379 & 4504 & 1130.2 & 66.3 & 38.1 & 1234.6 & 49.2 & 41.6 & 784.0 & 35.1 & 3.8 & 89.8 & & \\
\hline & Nov & 4670 & 5602 & 1439.2 & 110.8 & 59.6 & 1609.6 & 99.7 & 56.3 & 892.0 & 55.8 & 7.5 & 136.5 & & \\
\hline & $\operatorname{Dec}^{6}$ & 3877 & 3795 & 1019.7 & 100.5 & 44.2 & 1164.4 & 54.7 & 25.3 & 511.5 & 16.3 & 1.3 & 55.9 & 1420.7 & 158.7 \\
\hline
\end{tabular}

Note: some figures have seen small revisions since they were last published.

1 Data are available only quarterly and in the months following the quarter.

2 Percentage alcohol by volume.

3 Made wine with alcoholic strength $1.2 \%$ to $5.5 \%$, includes alcoholic lemonade of appropriate strength.

4 From 28 April 2002 duty on spirit-based ready-to-drink (RTDs) products is charged at the same rate as spirits per litre of alcohol.

Spirit-based RTDs were previously dutied at the made wine rate and details on quantities can be found in made wine coolers.

5 Includes imported spirits.

6 Due to the effect of the holiday period, these figures are subject to greater uncertainty than usual. Also, unusually high or low figures

may be changed on receipt of amendments to returns data.

7 Provisional.

8 Revisions are possible from July 2010.

Source: HM Revenue and Customs Alcohols Bulletins at https://www.uktradeinfo.com/index.cfm?task=bullAlcohol 


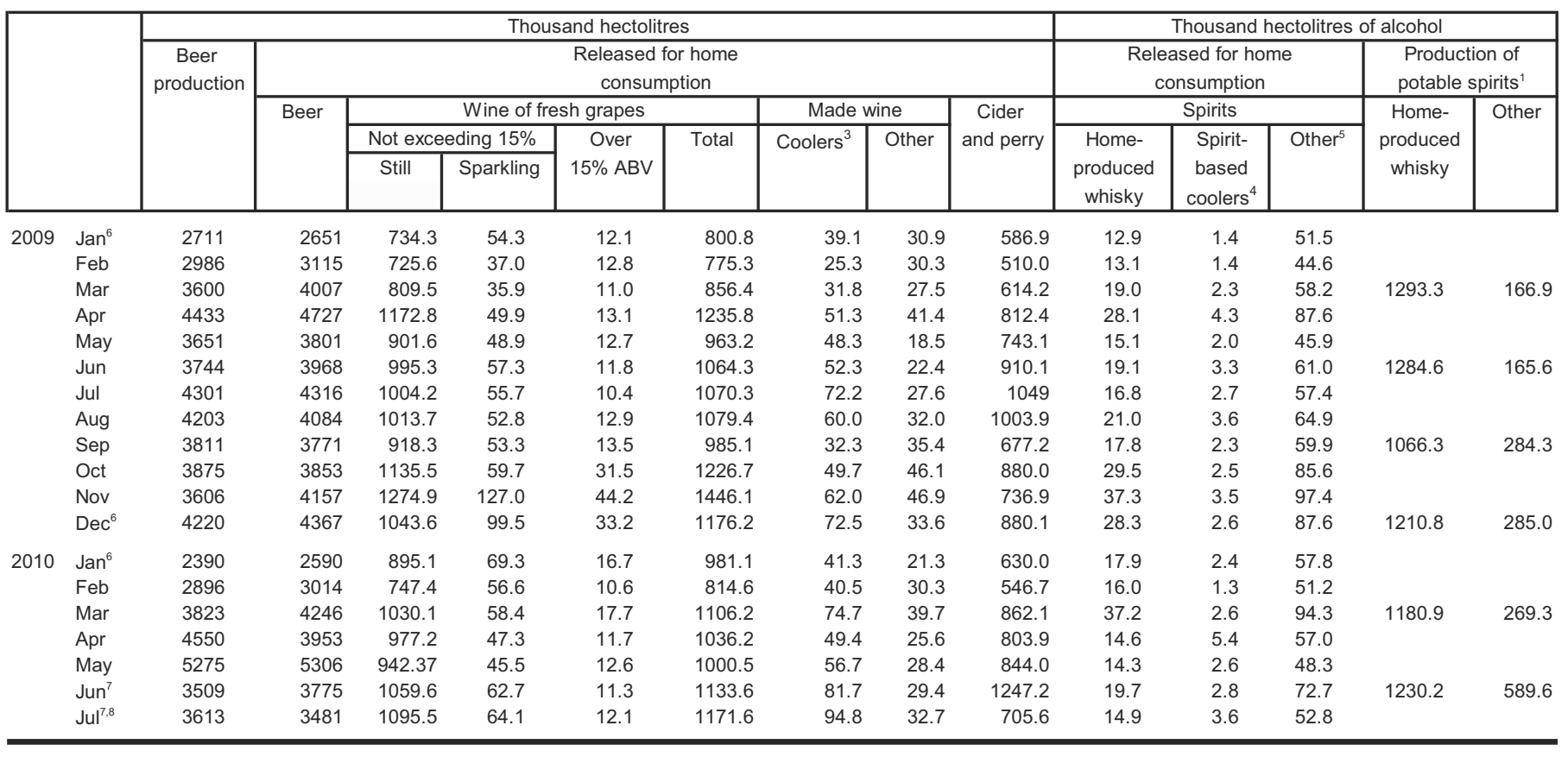

See notes on first page of table 


\section{Table 7.8}

Index numbers of textile and clothing industries

Standard Industrial Classification 2003

$2005=100$, seasonally adjusted

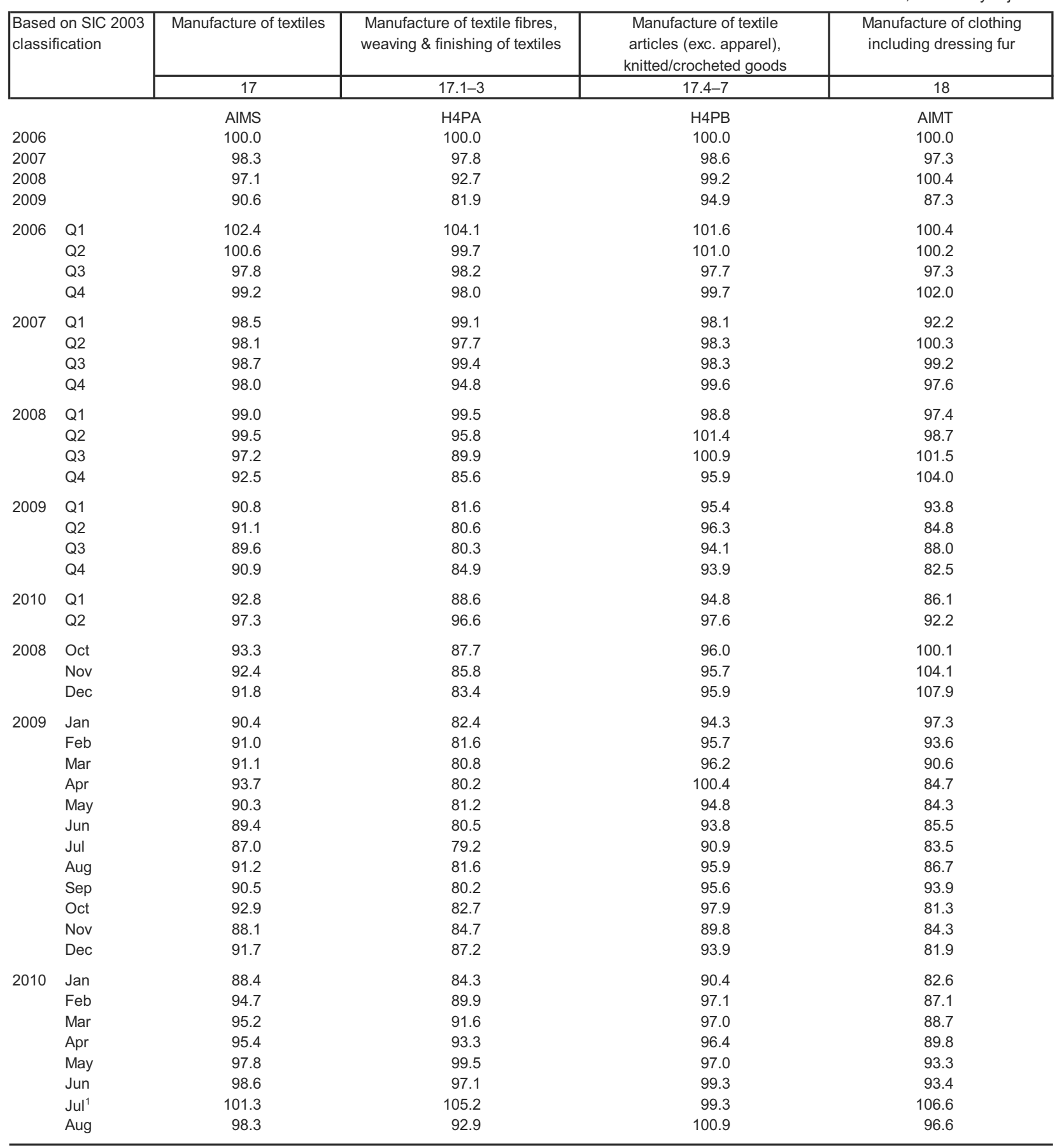

Note: ONS has introduced metholological changes to the Index of Production from March 2008 (January 2008 data). Also, a new industry structure means that the level of detail published is reduced. For the textile and clothing sector, industries are no longer available at the full level of disaggregation. Further information can be found on the NS website at - http://www.statistics.gov.uk/cci/article.asp?id=1908.

1 Revisions are possible from July 2010

Source: Tel: 01633455803 